\title{
Del offline a la r@dio: las experiencias de la industria radiofónica española y colombiana.
}

\section{From offline to r@dio: the experiences of the Spanish and Colombian radio industries.}

\author{
Gutiérrez-García, M. y Barrios-Rubio, A. ${ }^{1}$ \\ Recibido: 20-08-2018 - Aceptado: 15-01-2019 \\ DOI: https://doi.org/10.26441/RC18.1-2019-A4
}

RESUMEN: En el proceso de adaptación de la industria radiofónica a la naturaleza del entorno digital, los operadores implementan estrategias que vehiculan la convivencia del offline, todavía core del negocio, y el online en aras a definir su proyecto de r@dio. El objetivo de este artículo es determinar qué tipo de actividades desarrolla, su grado de eficacia y hasta qué punto influyen las particularidades de su propio mercado. La investigación se centra en dos escenarios, España y Colombia, y plantea un abordaje metodológicamente mixto, a partir del diseño de instrumentos que dan insumos cuantitativos, sin dejar de lado elementos cualitativos, de lo que acontece en los perfiles de las redes sociales y las web-r@dio de las emisoras. Como conclusiones, se observa que la difusión crossradial parte del sonido offline que en el marco de la táctica $360^{\circ}$ se vuelve testimonial frente a la presencia de texto, fotografías y vídeos.

Palabras Clave: radio; difusión crossradial; redes sociales; narrativa transradiofónica; ecosistema digital.

\begin{abstract}
In the process of adaptation of the radio industry to the nature of the digital environment, operators are implementing strategies that convey the coexistence of the offline, still as a core business, and the online, in pursuit of the definition of their own r@dio project. The objective of this article is to determine the type of activities that are developed, their efficiency and influence in the peculiarities of their own market. Research is carried out in two different scenarios, Spain and Colombia, and it poses a methodologically mixed approach from the design of instruments that provide quantitative inputs. It is also important to bear in mind the qualitative elements of the events of social media and the ones from web radio. As a conclusion, it is observed Broadcasting comes from the offline sound, which in the context of the $360^{\circ}$ tactic, becomes a testimony in the presence of text, photographs and videos.
\end{abstract}

Keywords: radio; radio broadcasting; social media; radio narrative; digital ecosystem.

1 Maria Gutiérrez-García es Doctora en Ciencias de la Información Sección Periodismo, Profesora Titular y Directora del Departamento de Comunicación Audiovisual y Publicidad de la Universidad Autónoma de Barcelona. maria.gutierrez@uab.cat, http://orcid.org/0000-0002-2340-7677

Andrés Barrios-Rubio es Doctor en Contenidos de Comunicación en la Era Digital de la UAB, Profesor Titular y Director del Departamento de Comunicación social y cinematografía de la Universidad de Bogotá Jorge Tadeo Lozano. andres.barrios@utadeo.edu.co, http://orcid.org/0000-0001-9838-779X 


\section{Introducción}

Los paradigmas que generan, conceptual y operativamente para la industria radiofónica, la sociedad del conocimiento, las tecnologías de la información y la comunicación, y la globalización, rodean el ecosistema mediático con su infraestructura y aplicaciones que dan respuesta a la oferta y demanda de servicios por parte de los usuarios. Si bien los medios convencionales continúan siendo el eje central de la mediación social (difusión de información, propagación del conocimiento, impulso democrático), no se puede obviar que los medios online y las plataformas de comunicación han entrado en el panorama mediático reconfigurando la concepción de los mass media convencionales, sus rutinas productivas y modelo de negocio. Convergencia de antena y dispositivos de pantalla que trae consigo un nuevo escenario de acción y distribución sonora -streaming, podcasts- $\mathrm{e}$ interacción con la audiencia dando origen a lo que esta investigación ha denominado como r@dio (Barrios \& Gutiérrez，2016b; Barrios-Rubio \& Gutiérrez-García, 2017).

El acoplamiento de medios, la relación medio-usuario, las dinámicas de mercado, el impacto de las redes sociales y el fenómeno de la convergencia, son los temas que mayoritariamente han suscitado el interés de los investigadores por abordar el estudio de caso desde diversas perspectivas y escenarios. El determinar los usos y consumos de las web-r@dio (Barrios-Rubio \& Gutiérrez-García, 2017), y la formación de microredes de acción permiten de- terminar qué está haciendo la industria radiofónica, española y colombiana, para adaptarse a los nuevos entornos digitales. Es esta una dimensión comunicativa en la que se establece un nuevo modelo de comunicación y narrativas cambiantes que trascienden del transistor a los dispositivos de pantalla. Así, desde la perspectiva de la gestión de la información se ha apostado por captar la atención de nuevos públicos, usuarios con coordenadas espacio-temporales divergentes a las tradicionales.

\section{Marco teórico: la radio offline construye un entorno en línea}

La industria radiofónica ha implementado su presencia en Internet sumida en una crisis económica, consecuencia de la baja penetración en el segmento juvenil y del descenso de la inversión publicitaria, que ha puesto en cuestión el modelo de negocio (Martí et. al., 2015; Vara-Miguel \& DíazEspina, 2015; Afuah, 2014, McNamara,2010) al tiempo que se emergían nuevas dinámicas de consumo (Bonini et. al., 2016; Monclús et. al., 2015). Sin embargo, la industria ha partido del "concepto tradicional de radio y de sus características para aliarse con el potencial de la red' (Reis, A.I., 2015:23), poniendo énfasis en la web y en las redes sociales (Cabrera, 2016) para sumergirse progresivamente en tácticas de difusión crossmediáticas y explorar narrativas transradiofónicas (MartínezCosta, 2015; Bechmann, 2009; Jenkins, 2006; Mittel, 2006; Aarseth, 2006; Bechmann, 2006; Boumans, 2004) en aras a definir un proyecto de r@dio 
(Barrios-Rubio \& Gutiérrez-García, 2017). Desde esta perspectiva, la mayoría de proposiciones se han desarrollado con la confianza, entre otros, de detener la baja presencia del medio tradicional en la dieta mediática juvenil, una situación que ha afectado a la mayoría de los mercados radiofónicos. Así, lo pusieron de manifiesto estudios de audiencia de referencia como Radio Joint Audience Research -RAJAR(U.K), Médiamétrie (Francia), Estudio General de Medios -EGM- (España), etc., pero también informes sobre el sector publicados por organizaciones como la Oficina de Comunicaciones del Reino Unido -OFCOM- y la Unión Europea de Radiodifusión -UER-, entre otros, que alertaron a la industria sobre el freno en la penetración del medio entre los jóvenes de 14 a 24 aproximadamente, consecuencia del efecto seductor del entorno digital especialmente entre los jóvenes de 14 a 24 años. En este sentido, son numerosos los estudios que avalan que la presencia en las redes sociales, por una parte, ha facilitado la localización de usuarios que, jóvenes o no, habían abandonado la emisión analógica y, por otra, ha impulsado y reforzado los vínculos con la audiencia (Stark \& Weichselbaum, 2013; González Molina \& Ramos del Cano, 2013; Herrera-Damas \& RequejoAlemán, 2011). Aunque esto último continúa siendo fundamental, informes de diferente índole así lo apuntan, lo cierto es que el consumo radiofónico analógico continúa decreciendo especialmente entre los jóvenes (EBU, 2016) del mismo modo que el tiempo medio de exposición, una situación que queda refrendada por los estudios de audiencia nacional como EGM (España) y ECAR (Colombia).

El despliegue de la industria radiofónica en el entorno online comportaba modificar el modelo de comunicación, primero, pasando de lo efímero del mensaje sonoro a la perdurabilidad mediante el podcast, los archivos de vídeo, y los mensajes y textos que circulan en la Web y las plataformas de comunicación y, segundo, poniendo al oyente en el epicentro de la estrategia. Ello suponía asumir la pérdida de la verticalidad en la interacción con la audiencia al eludir el control del emisor (Barrios-Rubio, 2013). Pese a ello, el rol de los networked listeners (Bonini et alt. 2016) continúa condicionado por las pautas que impone la antena (Pinseler, 2015; Piñeiro Otero, 2015; Gutiérrez, et al., 2014; Bonini \& Sellas 2014; Ramos del Cano, 2014). Aunque se observen cambios en aras a la construcción de 'una interacción real' (Centereno de Arce, 2016:192), la falta de reconocimiento en la antena (Monclús, et al., 2015) refuerza la idea de que la presencia en las redes tiene básicamente un objetivo estratégico, difícil de monetizar (Clemons, 2009), que se fundamenta principalmente en el incremento de la distribución de contenidos (Edmond, 2014).

Según RAJAR (2016) y OFCOM (2015) los social media se han convertido para los jóvenes británicos de 15 a 24 años en un modo de consumir información. En España, el II Estudio Anual Audio Online (2017) concluye que un 
$47 \%$ de los internautas oye radio online en directo y un $43 \%$ en diferido y, en Colombia, IBOPE (2017) evidencia que el $49 \%$ escucha radio por Internet, porcentajes que corresponden a la audición de emisoras analógicas en la red y que por el momento superan a las puramente online. De hecho, 'siguen siendo los medios tradicionales quienes logran el mayor número de seguidores y tienen mayor capacidad de influencia sobre los mismos, dado que en la red se autorefuerzan los hábitos de consumo' (Gutiérrez-Coba, et. al., 2012 en Barrios-Rubio, 2016:127) además de la 'fidelización de los oyentes' (Fontdevila, \& Lamelo, 2015:814).

Situar a la audiencia en el epicentro de las acciones industriales implica explorar estrategias de vinculación emocional que, por una parte, se desplegarán en términos de afecto y cumplimiento de sus expectativas (Hennig-Thurau, et. al., 2010; Best, 2007) y, por otra, gracias a las plataformas facilitarán la interconexión entre usuarios para comentar y compartir (Chan \& Ngai, 2011). En definitiva, se propicia la viralidad de contenidos radiofónicos 'generando recomendaciones entre consumidores' (Castelló, 2010:94) con el objetivo de poner en valor la reputación de la marca (Del Pino, Castelló \& Ramos Soler, 2013), especialmente en el entorno digital. Desde esta óptica, debe recordarse que en general la industria radiofónica ha implementado proyectos que partían del offline y consecuentemente la imagen de marca ha continuado asociada a la antena.

\section{Metodología}

Este artículo tiene como objetivo analizar de qué modo los operadores radiofónicos utilizan las redes sociales para posicionar en el epicentro de sus estrategias el consumo de contenidos centralizados en las web-r@dio a partir del análisis de caso de la radio privada española y colombiana. Partiendo de la premisa de que cada operador asociado a una marca desarrolla una hoja de ruta acorde con el mercado offline en el que compite y que las redes sociales facilitan la extensión de su área de influencia en el online, se buscará dar respuesta a las siguientes preguntas:

QR.1. ¿Es la convergencia antenaecosistema digital la estrategia principal implementada por la industria en la construccióndela r@dio?

QR.2. ¿La estrategia digital de la industria radiofónica estimula el consumo de contenidos desde y para diferentes plataformas, ya sea redes sociales o web-r@dio?

QR.3. ¿Las estrategias implementadas están influenciadas por el ámbito de difusión offline, es decir, el mercado propio?

La estructura de los mercados radiofónicos en ambos países es básicamente diferente. En España, está conformada por un sector público que aglutina 1 grupo estatal (RTVE) con 5 redes de difusión y 13 grupos públicos regionales con 25 cadenas. Aunque la oferta básica es la generalista, también hay presencia de canales musicales y, en algunos casos, all news. En el ámbito privado, son 3 los conglomerados dominantes (Prisa Radio, Grupo COPE y 
Uniprex), representados aproximadamente por 13 grupos que ofrecen tanto contenido de radio generalista como temática musical, aunque también se observan grupos de menor calado dado que no cuentan con el suficiente número de frecuencias como para difundir su oferta por todo el territorio español (Badillo \& Pérez Alaejos, 2012). En relación a la audiencia, la incidencia de la radio privada es significativamente superior que el de la pública en todos los ámbitos $(76 \%$ en la oferta generalista y $95 \%$ en la temática musical) con la excepción de la temática informativa, ámbito en el que la pública alcanza el 50,6\% (AIMC, 2018). Conviene resaltar que la radio privada también cuenta con grupos de ámbito analógico regional. Un caso paradigmático es el de la generalista RAC 1 que opera en Cataluña y ha conseguido en los últimos estudios de audiencia situarse entre las 5 primeras. El número de radios municipales y de locales privadas es fluctuante dado que algunas de ellas han ido desapareciendo como consecuencia de la crisis económica dando lugar a otras nuevas. A todo ello, se le suma un ingente volumen de emisoras del tercer sector, todavía sin regulación, y otras que están operando sin licencia (Niqui \& Segarra, 2016), dando lugar a un espectro radiofónico saturado.

En el ecosistema mediático colombiano, la radio pública congrega a RTVC como grupo estatal con 2 redes de difusión en antena (55 emisoras generalistas, 9 temáticas) que alcanzan el $1 \%$ de la sintonía nacional. Las emiso- ras privadas comerciales, con una penetración del 97\%, tienen su mayor representación en tres cadenas radiales (Caracol, RCN y BLU) que cuentan con emisoras generalistas y temáticas musicales articuladas en 8 grupos radiales minoritarios y emisoras independientes de baja penetración en los ranking de audiencia (Barrios-Rubio, 2016). El mercado radiofónico colombiano lo complementan las estaciones radiofónicas comunitarias, así como las emisoras de las fuerzas públicas (Ejercito y Policía) y las universidades. Esta esfera sonora está integrada por 1578 radiodifusoras y alcanza una penetración del $83 \%$ del mercado mediático de Colombia.

Teniendo en cuenta que el core del negocio radiofónico continúa situado en el ámbito analógico (Barrios \& Gutiérrez, 2016; Martí, et. al., 2015), la muestra se ha construido a partir de los índices de audiencia de las emisoras generalistas publicados por las entidades homologadas en cada país. Si bien es cierto que la radio temática musical aglutina un mayor volumen de audiencia, en torno al 57\% según el EGM y al $77 \%$ según ECAR, la radio generalista es la que despierta un mayor interés entre los grupos mediáticos a la hora de invertir (Barrios-Rubio, 2016) en parte por su capacidad de crear opinión pública. Como datos complementarios, cabe destacar que del total de oyentes que consumen radio temática en España tan sólo el 7\% lo es de radio informativa, mientras que en Colombia el $2 \%$ consumen marcas deportivas y religiosas. 
La muestra (Tabla 1) corresponde a las webs corporativas y perfiles en Facebook y Twitter de las tres cadenas privadas más escuchadas en cada uno de los mercados analizados: las españolas Ser, grupo Prisa Radio, (4.269.000 oyentes diarios), Cope (2.588.000) y Onda Cero, grupo Uniprex, (1.767.000) y las colombianas Caracol Radio (1.749.000), Blu Radio (1.117.000) y W Radio (1.089.000). La recolección de la muestra se efectuó bajo el criterio de semana compuesta (Lozano, 1994; Redondo, 2007; Barrios \& Gutiérrez, 2016) durante el mes de noviembre de 2016.

Tabla 1. Muestra objeto de estudio

\begin{tabular}{|c|l|l|l|l|}
\hline País & \multicolumn{1}{|c|}{ Emisora } & \multicolumn{1}{|c|}{ URL } & \multicolumn{1}{c|}{ Cuenta Twitter } & \multicolumn{1}{|c|}{ Perfil Facebook } \\
\hline \multirow{4}{*}{ España } & Ser & http://www.cadenaser.com & @La_SER & /cadenaser \\
\cline { 2 - 6 } & Cope & http://www.cope.es & @ cope_es & /cope \\
\cline { 2 - 6 } & Onda Cero & http://www.ondacero.es & @OndaCero_es & /ondacero \\
\hline \multirow{4}{*}{ Colombia } & Caracol Radio & http://www.caracol.com.co & @CaracolRadio & /caracolradio \\
\cline { 2 - 6 } & Blu Radio & http://www.bluradio.com & @BluRadioCo & /BluradioColombia \\
\cline { 2 - 6 } & W Radio & http://www.wradio.com.co & @WradioColombia & /WRadioCo \\
\hline
\end{tabular}

Fuente. Elaboración propia.

* Caracol Radio y W Radio son emisoras de la Cadena Radial Colombiana que pertenece al Grupo Prisa de España (Cadena Ser), pero actúan de modo independiente.

Se diseñó una herramienta metodológica que combinaba datos cuantitativos que facilitaban un abordaje cualitativo del objeto de estudio (Hernández, Fernández \& Baptista, 2003). Para garantizar la integralidad del análisis, se sometió a pruebas de fiabilidad (Wimmer \& Dominick, 1996) acorde con los coeficientes intercodificadores de Holsti (1969) alcanzándose un grado de coincidencia del $97 \%$, es decir, un margen de error del 3\%. El proceso sirvió para eliminar cualquier tipo de sesgo que pudiera presentar la herramienta científica (Orteha \& Galhardi, 2013). El corpus y las categorías de análisis se sintetizan en la Tabla 2.

La diversidad de ecosistemas permite observar también si el entorno offline influye en las estrategias y patrones que los operadores implementan en las propias marcas. El análisis visualizará hasta qué punto los factores locales inciden en un fenómeno que a priori es global. 
Tabla 2. Categorías de análisis empleadas en el desarrollo investigativo

\begin{tabular}{|c|c|c|c|c|}
\hline Plataforma & $\begin{array}{l}\text { Método de } \\
\text { recolección }\end{array}$ & $\begin{array}{l}\text { Muestra } \\
\text { recogida }\end{array}$ & $\begin{array}{l}\text { Categorías de } \\
\text { análisis }\end{array}$ & $\begin{array}{l}\text { Descripción de la } \\
\text { categoría }\end{array}$ \\
\hline \multirow{3}{*}{ Twitter } & \multirow{3}{*}{$\begin{array}{l}\text { Mensajes del } \\
\text { microblogging } \\
\text { Twitter almacenados } \\
\text { a través del portal } \\
\text { Twitonomy, que } \\
\text { suministra la } \\
\text { actividad de la } \\
\text { cuenta y permite su } \\
\text { descarga con } \\
\text { indicadores de uso y } \\
\text { respuesta. }\end{array}$} & \multirow{3}{*}{5658 tuits } & $\begin{array}{l}\text { Actividades de } \\
\text { producción de la } \\
\text { industria } \\
\text { radiofónica }\end{array}$ & $\begin{array}{l}\text { Número de publicaciones, } \\
\text { hora de uso, tipo- } \\
\text { intencionalidad del } \\
\text { mensaje. }\end{array}$ \\
\hline & & & $\begin{array}{l}\text { Correlación del } \\
\text { mensaje con el } \\
\text { contenido }\end{array}$ & $\begin{array}{l}\text { Contenido del mensaje, tipo } \\
\text { de guía que se brinda al } \\
\text { receptor. }\end{array}$ \\
\hline & & & $\begin{array}{l}\text { Respuesta del } \\
\text { usuario }\end{array}$ & $\begin{array}{l}\text { Reconocimiento e } \\
\text { interacción que se brinda a } \\
\text { los mensajes del emisor. }\end{array}$ \\
\hline \multirow{3}{*}{ Facebook } & \multirow{3}{*}{$\begin{array}{l}\text { Publicaciones de } \\
\text { Facebook extraídas } \\
\text { directamente del } \\
\text { Fanpage de la } \\
\text { emisora, mediante } \\
\text { la captura de } \\
\text { imágenes. } \\
\text { Información se } \\
\text { complementada con } \\
\text { el análisis } \\
\text { estadístico de } \\
\text { LikeAlizer. }\end{array}$} & \multirow{3}{*}{$\begin{array}{l}2580 \\
\text { posts }\end{array}$} & $\begin{array}{l}\text { Actividades de } \\
\text { producción de la } \\
\text { industria } \\
\text { radiofónica }\end{array}$ & $\begin{array}{l}\text { Número de publicaciones, } \\
\text { hora de uso, tipo- } \\
\text { intencionalidad del } \\
\text { mensaje. }\end{array}$ \\
\hline & & & $\begin{array}{l}\text { Correlación del } \\
\text { mensaje con el } \\
\text { contenido }\end{array}$ & $\begin{array}{l}\text { Contenido del mensaje, tipo } \\
\text { de guía que se brinda al } \\
\text { receptor. }\end{array}$ \\
\hline & & & $\begin{array}{l}\text { Respuesta del } \\
\text { usuario }\end{array}$ & $\begin{array}{l}\text { Reconocimiento e } \\
\text { interacción que se brinda a } \\
\text { los mensajes del emisor. }\end{array}$ \\
\hline \multirow{3}{*}{ Web-radio } & \multirow{3}{*}{$\begin{array}{l}\text { Páginas web de } \\
\text { cada emisora } \\
\text { capturadas el día de } \\
\text { análisis en horario } \\
\text { aleatorio. }\end{array}$} & \multirow{3}{*}{$\begin{array}{l}30 \\
\text { páginas } \\
\text { web }\end{array}$} & Línea de guía & $\begin{array}{l}\text { Composición visual, mapa } \\
\text { de contenido, herramientas } \\
\text { iconográficas. }\end{array}$ \\
\hline & & & $\begin{array}{l}\text { Componente } \\
\text { informativo }\end{array}$ & $\begin{array}{l}\text { Propuesta temática que } \\
\text { desarrolla el emisor. }\end{array}$ \\
\hline & & & $\begin{array}{l}\text { Contenido } \\
\text { multimedia }\end{array}$ & $\begin{array}{l}\text { Alternativas exclusivas para } \\
\text { la web, convergencia } \\
\text { audiovisual, fotográfica, } \\
\text { sonora (narración } \\
\text { transradiofónica). }\end{array}$ \\
\hline
\end{tabular}

Fuente. Elaboración propia.

\section{Resultados}

\section{1. Twitter, la r@dio en 140 caracte-} res

Las cadenas colombianas mantienen en la red social Twitter un mayor flujo de actividad que las españolas, quizás como consecuencia de contar con un número más elevado de seguidores (Tabla 3). En este punto, es importante recordar que en los perfiles corporativos colombianos converge el conjunto de la programación, mientras que en España cada programa además cuenta con el suyo propio. 
Tabla 3. Datos generales de las cuentas corporativas de los medios en Twitter

\begin{tabular}{|l|l|l|l|l|l|l|}
\hline \multicolumn{3}{|l|}{ España } & \multicolumn{3}{l|}{ Colombia } \\
\hline Cadena & Ser & Cope & Onda Cero & Caracol Radio & Blu Radio & W Radio \\
\hline Cuenta & @La_SER & @ cope_es & @OndaCero_es & @CaracolRadio & @BluRadioCo & @ WradioColombia \\
\hline Apertura & $\begin{array}{l}\text { Junio } \\
2009\end{array}$ & $\begin{array}{l}\text { Octubre } \\
2007\end{array}$ & $\begin{array}{l}\text { Septiembre } \\
2010\end{array}$ & Junio 2009 & Junio 2012 & Febrero 2009 \\
\hline $\begin{array}{l}\text { Seguidores } \\
\text { a Nov. 28 } \\
\text { de 2016 }\end{array}$ & 1.049 .231 & 288.319 & 436.360 & 2.941 .875 & 1.842 .155 & 2.969 .949 \\
\hline $\begin{array}{l}\text { Muestra de } \\
\text { tuits } \\
\text { analizados } \\
\text { en el } \\
\text { periodo de } \\
\text { estudio }\end{array}$ & 246 & 264 & 455 & 1.595 & 2.007 & 1.091 \\
\hline
\end{tabular}

Fuente. Datos extraídos directamente de las cuentas de Twitter de cada una de las emisoras objeto de estudio.

La observación de las actividades del emisor a lo largo del día ha puesto en evidencia que los operadores de ambos ecosistemas han concentrado buena parte de sus actividades entre las 06:00 a 10:00 horas (el 28\% para ambos ecosistemas), franja horaria de prime time en la que se ofertan los magazines buque insignia de cada estación, conducidos por periodistas y presentadores estrellas, y en los que se establecen las líneas editoriales de la estación radiofónica. Durante el resto de la jornada, el volumen de actividades ha fluctuado homogéneamente: de 10 a 12 horas (15\% España y 12\% Colombia); de 12 a 16 horas (23\% las españolas y $20 \%$ las colombianas); de 16 a 21 horas (22\% ambas) y, finalmente, de 21 a las 6 horas de la mañana (12\% España y $18 \%$ Colombia). Podría aventurarse que la estrategia de publicación ha estado en consonancia con la oferta de antena y el índice de audiencia. Así, los programas que han cumplido ambos requisitos han generado una mayor actividad del operador en la cuenta. Esta circunstancia también se ha detectado en el caso español donde los perfiles corporativos han competido con los del programa.

¿Qué tipo de estrategias implementan los operadores para situar a la audiencia en el centro de sus actividades? Dado que el mayor volumen de publicaciones se produce en el prime time, se ha focalizado el análisis en la franja de 06:00 a 10:00 de la mañana. A la luz de los datos, la primera constatación es que cada marca radiofónica ha perfilado un diferente rol en esta red social con independencia del mercado en el que compite. Así la cadena SER (73\%) y Caracol Radio (70\%) han mostrado un mayor interés por la circulación de noticias, seguidas a distancia por Onda Cero (30\%). En cambio, para W Radio 
(55\%) Cope (46\%) y Blu Radio (44\%) la promoción de asuntos ligados a la antena ha sido la variable a la que dedicó más tuits. El comportamiento detallado ha ratificado que había un importante porcentaje de retuits en los perfiles españoles, seguramente consecuencia de que la actividad digital se diversificó en cuentas corporativas y de programas promoviendo el RT entre estas. La colombiana Blu Radio ha seguido también esta estrategia de reforzamiento con la incorporación de periodistas de perfiles consolidados y con un significativo número de seguidores. Todo y esta coincidencia, en términos generales, las emisoras colombianas han cuidado con celo que la vinculación entre medio y usuario sea asociada a la marca radiofónica $(96 \%$ sobre el total de las publicaciones), mientras que las españolas han apostado más por perfiles de programas y personajes (44\% sobre el total de las publicaciones).

Una estrategia común ha sido la referencia a los perfiles de los personajes e instituciones, protagonistas de la información (18\% España, $14 \%$ Colombia). Además, la triangulación de dinámicas del ecosistema digital (web, Twitter y Facebook) ha propiciado el reconocimiento de los actores de la actualidad.Gracias al@ losnetworked listeners han accedido a la foto principal de la cuenta citada. Revisadas el $100 \%$ de las publicaciones emitidas por las emisoras, no se ha encontrado ninguna referencia a los perfiles de los usuarios, es decir, ha sido el medio quien ha direccionado el mensaje y quien ha ejercido la posición dominante en el modelo comunicativo.

El análisis de la muestra ha desvelado que estratégicamente las cadenas incorporaron en su contenido digital metabuscadores que las han ligado a las tendencias del día, pero también han propuesto hashtags (\#) para que los usuarios marcasen sus participaciones. Ello no solo ha ayudado a potenciar la marca del medio, sino que ha visibilizado la competencia digital de la emisora. En un alto porcentaje (67\% España, 76\% Colombia) los mensajes tenían agregado un hipervínculo que conducía al website de la emisora. Ello lleva a afirmar que se apostó por una táctica de consumo 360 que incorporó las dinámicas de antena al ecosistema digital, donde el usuario aparte del audio ha podido acceder a elementos textuales, iconográficos y visuales de fotografía y vídeo.

Sorprende que direccionar hacia documentos sonoros haya sido una estrategia de poco calado en general, dado que hubiera permitido rentabilizar los contenidos de la antena. De hecho, sólo W Radio (64\%) y Cope (51\%) la han seguido, aunque ha sido la primera la que ha apostado significativamente por el sonido, mientras que la segunda ha dado también preponderancia a la información textual acompañada de fotografía (46\%). En cambio, Ser (66\%), Blu Radio (66\%) y Caracol Radio (62\%) priorizaron el vídeo frente al audio. Con independencia de hacia dónde se haya conducido, el usuario ha reaccionado compartiendo el mensaje, marcándolo como favorito o comentándolo, acciones que han repercutido en el po- 
sicionamiento e impacto de la marca.

En relación al número de comentarios generados por cada publicación del emisor, Ser y Cope han resultado ser las más rentables seguidas de $\mathrm{W}$ Radio. Además, de las tres, la primera ha alcanzado el porcentaje más alto de retuits (59\%), lo que significó una mayor propagación de sus publicaciones. El índice de rentabilidad se obtuvo de dividir el total de reacciones de los usuarios entre el volumen de publicaciones del emisor (Ser 20, Cope 20, Onda Cero 6, Caracol 9, Blu Radio 11, W Radio 15).

$\mathrm{Al}$ margen de matices, el retuiteo ha aparecido como la actividad preferente del usuario en Twitter. En general, los comentarios presentaron una menor incidencia a excepción de Blu Radio (35\% sobre el total de tuits usuario) y W Radio (27\%). Sus significativos índices han evidenciado que, además de contar con un nutrido número de usuarios fieles a la marca, éstos han sido proactivos. Ahora bien, el análisis ha mostrado también un desinterés generalizado entre los emisores por estimular la interacción con los usuarios, habiéndose puesto de manifiesto la verticalidad en el proceso comunicativo y el bajo reconocimiento de estos (Pinseler, 2015; Ribes et al., 2015). La excepción poco significativa se ha localizado en Blu Radio y W Radio (ambas con un $2 \%$ sobre el total de publicaciones emisor), y Cope (1\%).

\section{2. Facebook: la promoción de la antena}

El impacto de Facebook, la red social con mayor expansión y uso por parte de los usuarios en España y Colombia, es menor en el conjunto de la muestra tanto por el número de amigos alcanzado como por el volumen de publicaciones del emisor (Tabla 4).

Tabla 4. Datos generales de los perfiles de los medios en Facebook

\begin{tabular}{|l|l|l|l|l|l|l|}
\hline & \multicolumn{7}{|l|}{ España } & \multicolumn{2}{l|}{ Colombia } \\
\hline Emisora & Ser & Cope & $\begin{array}{l}\text { Onda } \\
\text { Cero }\end{array}$ & $\begin{array}{l}\text { Caracol } \\
\text { Radio }\end{array}$ & Blu Radio & W Radio \\
\hline Cuenta & /cadenaser & /cope & /ondacero & /caracolradio & /BluradioColombia & WradioCo \\
\hline $\begin{array}{l}\text { Apertura } \\
\text { cuenta }\end{array}$ & 2009 & 2007 & 2010 & 2009 & 2012 & 2009 \\
\hline $\begin{array}{l}\text { Amigos a Nov. } \\
\text { 28 de 2016 }\end{array}$ & 457.964 & 132.599 & 226.224 & 580.039 & 832.949 & 213.759 \\
\hline $\begin{array}{l}\text { Publicaciones } \\
\text { del emisor } \\
\text { analizadas en } \\
\text { periodo de } \\
\text { estudio }\end{array}$ & 132 & 220 & 255 & 621 & 553 & 799 \\
\hline
\end{tabular}

Fuente. Datos extraídos directamente de los perfiles de Facebook de cada una de las emisoras objeto de estudio. 
El flujo de actividades tiende a ser estable a lo largo de la jornada, aunque los índices más altos se alcanzan en la franja prime time, momento en el que se ha concentrado el número más alto de publicaciones tanto del emisor como de los usuarios. Así, el roadmap ha estado en consonancia con la antena, una situación similar a la detectada en Twitter.

La mayoría de emisoras ha tendido a usar Facebook para promocionar contenidos de antena. En el caso español, este tipo de publicaciones ha destacado en tanto que son perfiles corporativos que también han establecido sinergias con la antena. Una excepción a tener en cuenta ha sido el significativo interés por anunciar podcasts de Onda Cero (37\% sobre el total de publicaciones), una tendencia seguida de cerca por Cope (20\%) y W Radio (18\%) y casi de forma testimonial por Blu Radio (4\%). Sin duda, ha sido una estrategia que, además de revitalizar los contenidos sonoros, ha estimulado el consumo asincrónico. La interacción con la audiencia y los posts compartidos se han convertido en singularidades de escaso seguimiento para la mayoría de los perfiles. Por el contrario, ha resultado singular la baja presencia de publicaciones destinadas a interactuar con los networked listeners (W Radio $7 \%$; Cope 4\%; y Blu Radio 2\%) y de posts compartidos (Ser 7\% y Blu Radio 2\%).

Completado el análisis, no se ha detectado autopromoción exclusiva de la emisora ni del programa en antena, solamente la etiquetación del contenido del podcast. Únicamente, Caracol Radio ha destinado el $100 \%$ de sus operaciones a informar de las noticias y entrevistas que tienen lugar en el ámbito offline. Desde una perspectiva cualitativa, se ha constatado el uso generalizado de hipervínculos que conducían al usuario hacia el website de la emisora. Así, el 6\% de las publicaciones españolas han empujado al usuario a otra red social de la emisora, especialmente a la del programa en cuestión. Por su parte, el $2 \%$ de las colombianas los han redirigido hacia perfiles de medios (radio o televisión) del propio grupo empresarial.

La mayor diferencia entre ambos ecosistemas se ha detectado en la táctica por marcar tendencia en la red promoviendo hashtags. Mientras en las emisoras españolas apenas se ha incidido en ello (6 de 162 publicaciones), en las colombianas (279 de 379 mensajes) ha constituido un mecanismo que ha ubicado al usuario en el acontecimiento (por temática y fuente) al tiempo que ha posicionado, propagado y fortalecido la marca radiofónica y sus programas.

A la luz de los datos, la industria radiofónica ha realizado acciones que han evidenciado ciertas modificaciones en sus rutinas productivas y una tendencia a la diversificación del modelo del negocio. Por una parte, el audio ha reivindicado su protagonismo con los podcasts (19\% de la muestra analizada en España, $7 \%$ en Colombia), aunque ha seguido relegado frente a productos multimedia que han apostado por narrativas transradiofónicas (65\% de los mensajes españoles, $83 \%$ colombianos). Por otra parte, se ha observado también un incipiente uso de Facebook Live (16\% emisoras españolas, $10 \%$ colombianas) generando vídeos cuyo número de reproducciones 
ha resultado ser significativo entre los networked listeners del prime time: Ser 80.868, Cope 202.452, Onda Cero 20.163, Caracol Radio 17.233, Blu Radio 127.832. W Radio ha sido la única en no emplear esta estrategia en el prime time, aunque sí lo ha explorado en otras franjas horarias. En la actualidad, transmite en Facebook Live un programa de 90 minutos en la franja del mediodía.

En las redes, ha sido importante el grado de compromiso obtenido al dividir el total de reacciones de los usuarios entre el volumen de publicaciones del emisor (Ser -28\%-, Cope-65\%-, Onda Cero14\%-, Caracol Radio -15\%-, Blu Radio -21\%-, W Radio -7\%-) que, hasta cierto punto, ha evidenciado lo constante de esa relación. Así, Cope ha conseguido un significativo porcentaje con acciones puntuales de difusión crossmediática y narrativas transradiofónicas soportadas en el vídeo. Desde esta perspectiva, la fidelización de los usuarios de Facebook con el contenido de las emisoras españolas ha sido mayor (36\%) que el alcanzado por las colombianas (14\%). Ello se ha certificado con la cantidad de "Me Gusta" (69\% de los mensajes españoles, $58 \%$ colombianos), comentarios (9\% España, 20\% Colombia) y el número de veces que una publicación ha sido compartida (22\% emisoras españolas y colombianas).

\section{3. El website como escenario}

\section{convergente de la industria radiofónica}

La homogeneización formal tanto en lo referente a la información sobre la antena como en el acceso al streaming de dicha señal ha sido una tendencia generalizada. Quizás, la voluntad de brindar al usuario una guía básica para el consumo de la marca haya sido la clave para entender la coincidencia en la categorización del contenido, la disposición de etiquetas para el consumo a la carta, los blogs, el ámbito multimedia y las propuestas del emisor. Si bien ha variado la forma de nombrarlo, el patrón prácticamente ha resultado ser el mismo.

La similitud en las cabeceras se ha reforzado internamente en el caso de SER, Caracol Radio y W Radio, cadenas que pertenecen a un mismo grupo empresarial (Prisa). Estas han mantenido esquemas estratégicos parejos que se han adaptado a los colores distintivos de cada emisora, y a las características particulares de la idiosincrasia española y colombiana. Si bien estos elementos comunes se han dispuesto de modo diferente a lo largo del scroll del home, puede aventurarse que el principal objetivo de los websites analizados ha sido destacar la actualidad e inmediatez, características intrínsecas a la radio offline. De este modo, la marca radiofónica se ha constituido en un portal informativo en el que han sido mostrados los eventos más destacados del país.

La estructura de presentación se ha organizado en ventanas en las que se han articulado los temas objeto de interés periodístico con atractivos titulares y leads invitando a ampliar la información. Habitualmente se han acompañado de una foto y han podido incorporar audio y algún contenido multimedia. En general, el material presente en la web ha concordado con las propuestas de hipervínculos que, al igual que en las redes sociales, sugerían un recorrido para 
la exploración del tema. La incidencia de la antena sobre los contenidos web en ambos ecosistemas radiofónicos se reflejó en la relevancia que se le ha dado a la opinión de directores y/o periodistas del medio y significativos colaboradores.

En el entorno web, además de replicar el contenido offline, también se ha ofrecido propuestas sonoras complementarias y diferentes al contenido de antena. El catálogo ha sido amplio y variopinto, yendo desde los retazos de entrevistas a programas completos e incluso extras, es decir, producidos solo para el online.

\section{Discusión}

El análisis ha demostrado que, con independencia del ecosistema al que pertenezcan, las acciones estratégicas de la r@dio dependen básicamente de los intereses y expectativas de la empresa radiofónica en relación a la marca. De hecho, algunas de las coincidencias detectadas entre Ser y Caracol Radio han sido más consecuencia de su categoría como líderes de audiencia que de pertenecer a un mismo conglomerado empresarial (Prisa Radio). Sólo así puede interpretarse que W Radio, aún perteneciendo a Prisa, haya seguido diferentes directrices de sus homólogas. A la luz de los datos, se puede afirmar que la filosofía de la marca offline se ha extendido hacia el entorno online, primero, por la alta presencia de avances y resúmenes de lo acaecido en la antena y, segundo, por el especial énfasis en aquellos asuntos de carácter informativo que han construido el relato del prime time.

La concentración de las actividades en el prime time fue un indicativo de que to- davía la industria no ha entendido el potencial de las redes sociales que continúan siendo vistas como vías de propagación y no como espacios que propician nuevos modos de consumir, pero también de producir de contenidos. De hecho, el seguimiento de las dinámicas del offline obvió que los usuarios son particularmente activos durante la noche (MD. Marketing directo, 2017; Contreras; 2017), circunstancia que sin duda ha dificultado el encuentro entre un emisor que ha continuado viendo al usuario como un oyente que consume el programa en simultaneidad con la emisión. Por lo general, la puesta en escena se ha limitado a la publicación de texto acompañado de fotografía y quizás vídeo, pero con una minoritaria presencia de audio. En contraste, la irrupción de Facebook Live debe ser valorada de modo exitoso dado el número de reproducciones que ha alcanzado. La insistencia en imponer imágenes al sonido se ha convertido en una tendencia aceptada por la mayoría de los operadores. Si bien es todavía pronto para evaluar su desarrollo y repercusión en el consumo, dicha estrategia deberá ser tenida en cuenta en próximas investigaciones.

Sorprendentemente desde una perspectiva cualitativa, los networked listeners han despertado poco interés dada la ausencia de estrategias que destinadas a estimular la interacción y romper la verticalidad del offline en las redes sociales. Así, el emisor se ha limitado a replicar los contenidos de antena esperando que el usuario actúe de distribuidor. De nuevo, se ha ratificado la existencia de un discurso propio en 
el entorno online donde el papel del emisor se ha circunscrito al de proveedor de información (Gutiérrez et. al., 2014). Sin desmerecer el valor que tiene el número de seguidores y amigos como referente del posicionamiento en Internet, la falta de reconocimiento de este nuevo oyente en la antena ha revelado la instauración de una política tendente a la obtención de resultados a corto plazo en una industria que, al menos hasta ahora, ha basado su operativo en la consolidación de un ideario de marca y en la fidelización de la audiencia. Este nuevo modo de actuar ha constatado la incertidumbre que todavía provoca el desconocimiento de los entresijos de la red y el modelo de negocio que de ello se derive.

Pese a la baja demanda de interacción, los usuarios han interactuado de diferente manera según la red y el ecosistema. Así, el análisis ha confirmado que los seguidores en Twitter de las emisoras colombianas han sido más propicios a publicar comentarios (Blu Radio $35 \%$ sobre el total de tuits; W Radio 27\%; Caracol 17\%) que los españoles (Onda Cero 13\%; Cope 9\%; Ser 8\%). Sin embargo, ambos han coincidido en elevar a la categoría de actividad preferente el RT (Ser 59\%; Onda Cero 48\%; Cope 45\%; Caracol 44\%; W Radio 43\%, Blu Radio 36\%). En relación a Facebook, se ha observado una baja tendencia a comentar las publicaciones del operador. La excepción ha estado protagonizada por W Radio que, con un índice de 34\%, se ha convertido en el perfil con más número de comentarios seguida a distancia por la española Onda Cero (14\%).
Los datos han evidenciado un bajo interés de los operadores por propiciar la interacción frente a una significativa inclinación a la promoción y/o difusión de lo que ha acontecido en la antena con resultados desiguales en cada uno de los perfiles. En este punto, debe recordarse que en las emisoras españolas conviven perfiles corporativos y de programas, que se retroalimentan. La valoración de las consecuencias de esta estrategia sin duda merece un estudio aparte desde la perspectiva de las sinergias y la recepción. Volviendo a los resultados del análisis, éste ha constatado que los protocolos de actuación en los distintos ecosistemas se asemejan desde la perspectiva del emisor, siendo las actividades de la audiencia las que han presentado algunos matices diferenciados.

Para la industria radiofónica, el website ha sido el espacio en el que convergen las acciones que se articularon desde las redes sociales. Como si de un escaparate se tratase, ha contenido todo aquello que desde la antena fue destacado, anunciado e informado en los perfiles de redes sociales, es decir, el site ha sido el lugar donde el usuario pudo consumir cualquiera de los contenidos producidos por la marca. Este entretejido de flujos ha apostado por fomentar un consumo de $360^{\circ}$ como alternativa para el reconocimiento de la marca radiofónica favoreciendo a la vez el paso del online al offline y viceversa.

Sin duda, la irrupción de la radio en Internet continúa provocando todavía cambios significativos en la concepción del medio. Así, se ha vuelto a poner en valor la inmediatez, una característica 
explotada en el ámbito analógico, especialmente en el abordaje de asuntos de carácter informativo, que también ha alcanzado al entorno digital. Probablemente esta circunstancia ha abierto un debate en las redacciones sobre si una noticia debe ser emitida primero en antena o publicada en el site y los social media. Sea cual sea la estrategia seguida, el análisis ha revelado que, en general, la información textual ha primado sobre el sonido, una circunstancia que sorprende dado que la antena continúa siendo el core del negocio. Si bien los contenidos on air han sido desgranados para convertirse en frases destacadas, la presencia del audio se ha reducido en la mayoría de los casos a fragmentos, fruto del reciclaje de la emisión. Esta operación ha requerido de un tiempo que pudo ralentizar la difusión por lo que, desde las redes sociales, la opción prioritaria ha sido redireccionar hacia textos y fotografías. Esta rutina productiva ha continuado ligada a la antena, pero ¿qué ocurre con la producción de contenidos sonoros extras, es decir, para las plataformas online? Si se tiene presente que la máxima actividad por parte del emisor se ha concentrado en la franja prime time, las posibilidades de localizar publicaciones anunciando la disponibilidad de este tipo de contenidos ha sido mínima. La estrecha relación con la antena, y por ende con la actualidad, hace que la producción de podcasts haya pasado desapercibida para los usuarios. Sin embargo, no puede negarse que las emisoras, unas más que otras, han invertido recursos en la proliferación de productos exclusivamente online que han pasado desapercibidos para aquellos usuarios que no han accedido directamente a la web.

\section{Conclusiones}

Esta investigación ha explorado las estrategias que empresas radiofónicas de oferta generalista pertenecientes a diferentes ecosistemas comunicativos, el español y el colombiano, están implementado en el ecosistema digital. El objetivo de enfrentarnos a dos ecosistemas comunicativos diferenciados, a dos realidades empresariales diferentes, obedecía la necesidad de verificar si la industria radiofónica seguía un protocolo común de actuación o bien era el mercado propio el que imponía sus dinámicas. Los criterios de selección de la muestra nos condujeron a fijar nuestra atención sobre la radio privada, tipo de radiodifusión que lidera los ránquines de audiencia, instrumento que valida su rentabilidad económica y social. A la luz de los datos, es el posicionamiento en los estudios de audiencia el que influye en el diseño de estrategias de convergencia que conduzcan a la construcción de la r@dio, un nuevo medio que combina la emisión de contenidos sonoros offline con la presencia en plataformas online y redes sociales, propiciando por una parte, el consumo asincrónico y, por otra, la interacción con la audiencia e incluso su participación como generador de contenidos.

Sin embargo, la antena todavía es el epicentro de la mayoría de las acciones que implementan los operadores. Si bien se han detectado experiencias 
emergentes de planteamientos transradiofónicos (Martínez-Costa, 2015), el mantenimiento del liderazgo en el ámbito de la antena convencional continúa pesando en la toma de decisiones (Martí et al., 2015). Esta situación no debe menoscabar el esfuerzo de la industria española y colombiana por reforzar la convergencia hacialar@dio a partirde sinergias que confluyen en roadmaps que buscan el desarrollo de acciones de 360 ‥ Así, la convergencia mediática que se propone desde los websites de las emisoras aspira por brindar un resumen temático de la información, particularizar la agenda informativa de cada región y las transmisiones sonoras producto de las desconexiones locales de las cadenas objeto de estudio. Aunque incipiente se han promovido acciones que buscan dinamizar la imagen y competencia digital de la marca invitando a formar parte de una experiencia de consumo crossradial que se inicia en la antena, se expande, promociona y visibiliza en las redes sociales y App de la estación radiofónica, de modo que se expande y concentra en el portal web.

Ahora bien, en los fundamentos de estas estrategias principalmente destaca un interés por extender la marca offline hacia el ecosistema digital. De ahí, la correlación entre los contenidos de antena y los online en las webs, una circunstancia que debería ser explorada ya que las condiciones de competencia en uno y otro ámbito son diferentes. En este sentido, la transformación en r@dio (BarriosRubio \& Gutiérrez-García, 2017) implica instaurar nuevas dinámicas de producción y gestión de contenidos. Todavía la mayoría de emisoras analizadas promueven un consumo bidireccional, de la antena a la web y viceversa. Pese a ello, el número de usuarios en sus perfiles Twitter y Facebook ha continuado creciendo (Tabla 5), lo que puede ser un indicio del interés de la audiencia por convertirse en actores proactivos que vayan más allá de opinar y comentar.

Tabla 5. Actualización de los datos generales de los perfiles de los medios en Twitter y Facebook

\begin{tabular}{|l|l|l|l|l|l|l|}
\hline \multicolumn{3}{|l|}{ España } & \multicolumn{5}{l|}{ Colombia } \\
\hline Cadena & Ser & Cope & Onda Cero & Caracol Radio & Blu Radio & W Radio \\
\hline Cuenta & @La_SER & @COPE & @ OndaCero_es & @ CaracolRadio & @ BluRadioCo & @ WradioColombia \\
\hline $\begin{array}{l}\text { Seguidores } \\
\text { Twitter a } \\
\text { Dic. 2 de } \\
\text { 2018 }\end{array}$ & 1.153 .311 & 353.423 & 504.538 & 3.394 .061 & 2.266 .202 & 3.595 .383 \\
\hline $\begin{array}{l}\text { Amigos } \\
\text { Facebook } \\
\text { a Dic. 2 } \\
\text { de 2018 }\end{array}$ & 626.221 & 176.773 & 258.317 & 786.879 & 1.626 .078 & 485.395 \\
\hline
\end{tabular}

Fuente. Datos extraídos directamente de los perfiles de cada una de las emisoras objeto de estudio. 
Sin embargo, tal como se desprende de la investigación la networked audience continua siendo la gran olvidada. Así, las redes sociales han seguido articulándose en la mayoría de los casos como escaparates de la producción radiofónica y no como espacios de interacción en los que emisor y usuarios se interpelen. Esta ausencia es especialmente significativa en la franja prime time, momento en que los operadores proponen la agenda político-social. Como el estudio ha demostrado la demanda de opinión ni es mayoritaria ni tampoco significativa entre las emisoras analizadas y cuando ésta se ha dado la presencia en la antena ha sido casi nula.

El estudio efectuado ha demostrado que las marcas continúan desarrollando estrategias que reflejan la rigidez del proceso comunicativo. En el análisis se ha apreciado que las pocas preguntas efectuadas a los usuarios han sido sondeos de opinión de poca o ninguna repercusión en la antena. El emisor, tras una publicación, ha tendido a obviar cualquier tipo de interacción que pudiese generarse. Así, los usuarios han creado su propio discurso, más o menos intenso según el mercado al que pertenece la emisora, siendo esta la única diferencia detectada a lo largo del estudio. En términos generales, los colombianos parecen más proclives a interactuar que los españoles. Sin embargo, esta evidencia debe ser interpretada como una ilustración de la situación ya que para alcanzar la categoría de conclusión debería realizarse un estudio específico sobre los comportamientos de la networked audience.
Las estrategias implementadas por la industria radiofónica en el ecosistema digital han respondido a una extensión de la marca radiofónica offline buscando reforzar sinergias con el online. Desde esta perspectiva, la convergencia del roadmap con la antena limita la potencialidad propia del entorno online dificultando el reencuentro de la marca con los jóvenes para quienes las redes sociales se han convertido en los espacios de consumo e interacción. Según RAJAR (2018), el $41 \%$ de los jóvenes entre 15 y 24 años han declarado recibir notificaciones de sus estaciones de radio favoritas a través de las redes sociales. Este tipo de datos, también de calado cualitativo, son necesarios para que la industria pueda diseñar estrategias encaminadas a contactar con este sector de la audiencia que básicamente se mueve en Internet. En este sentido y a modo de evidencia, es necesario resaltar el proyecto radiofónico $O h ! M y L O L$. La vida moderna de la cadena Ser con unos datos de audiencia analógica muy bajos pero con un excelente comportamiento en las redes sociales (AIM, 2018). La clave está en su lógica transradiofónica por la que el contenido es el epicentro de cualquier actividad comunicativa. De ahí que sea difundido por las redes sociales antes de llegar a la antena.

La investigación ha puesto en evidencia una correlación entre el roadmap y la antena. Así, lo que ha sido destacado en una también lo ha sido en la otra y consecuentemente el pico más alto de actividades justamente ha coincido con el prime time. Este comportamiento 
puede ser asociado al de un networked listener del programa de prime time, o lo que sería lo mismo al de un oyente que paralelamente también actúa como usuario. ¿Qué quiere decir esto? Que no hay un aprovechamiento del potencial de las redes y que el diseño de estrategias continúa partiendo de la ante- na. Así, en un futuro no muy lejano, la industria radiofónica deberá encontrar las claves que le permitan, a partir de la interpretación de las dinámicas de consumo de su audiencia, diseñar estrategias articuladas que atienda la lógica de la narración transradiofónica y la difusión crossradiofónica.

\section{Bibliografía}

Aarseth, E. (2006). The culture and business of cross-media productions. Popular Communication, 4(3), 203-211.

AIMC - Asociación para la investigación de medios de comunicación. (2018). Resumen general. Año móvil Octubre 2017 a Mayo 2018. Madrid - España. Recuperado de https://www. aimc.es/a1mc-c0nt3nt/uploads/2018/06/resumegm218.pdf

AIMC (2016). Estudio General de Medios. EGM tercera ola 2016. Madrid - España. Recuperado de http://www.aimc.es/Nuevo-articulo,1881.html

Afuah, A. (2014). Business Model Innovation: Concepts, Analysis, and Cases. Routledge. New York: Routledge.

Badillo, Á. y Pérez Alaejos, M. (2012). Estructura del mercado radiofónico español. Transformaciones y tendencias del clivaje público/privado en el sistema radiofónico. En Gallego, J.I. y García Leiva, M.T. Sintonizando el futuro: radio y producción sonora en el siglo XXI, 61-104. Madrid: IORTVE.

Barrios-Rubio, A. (2013). La radio en la era de la sociedad digital. adComunica. Revista de Estrategias, Tendencias e Innovación en Comunicación. (5), 37-54. DOI: http://dx.doi. org/10.6035/2174-0992.2013.5.4

Barrios-Rubio, A. (2016). La radio generalista colombiana ante el desafío digital: un modelo en transición. Tesis inédita de Doctorado. Universitat Autònoma de Barcelona. Barcelona España. Recuperada de http://hdl.handle.net/10803/386494

Barrios, A. \& Gutiérrez, M. (2016). Transición y adaptación de la industria radiofónica colombiana privada al entorno digital desde la perspectiva de los operadores. Icono 14. 14 (2), 231-255. DOI: http://dx.doi.org/10.7195/ri14.v14i2.959

Barrios, A. \& Gutiérrez, M. (2016b). Migración de la estrategia radiofónica colombiana: del sonido a las pantallas sociales. Revista Latina de Comunicación Social, (71), 1243-1260. https://doi.org/10.4185/RLCS-2016-1144

Barrios-Rubio, A. \& Gutiérrez-García, M. (2017). Reconfiguración de las dinámicas de la industria radiofónica colombiana en el ecosistema digital. Cuadernosinfo, (41), 227-243. DOI: https://doi.org/10.7764/cdi.41.1146

Bechmann Petersen, A. (2006). Mediediffusion. Aarhus: The Centre for Internet Research. Bechmann Petersen, A. (2009). Crossmedia: Innovation Networks for Traditional Media Organizations. Tesis Doctoral. University of Aarhus. 
Best, R. J. (2007). Marketing estratégico. Pearson Educación: Madrid.

Bonini, T.; Caliandro, A. y Massarelli A. (2016). Understanding the value of networked publics in radio: employing digital methods and social network analysis to understand the Twitter publics of two Italian national radio stations. Information, Communication E Society, 2016, vol. 19(1), 40-58. DOI: http://dx.doi.org/10.1080/1369118X.2015.1093532

Bonini, T. \& Sellas, T. (2014). Twitter as a Public Service Medium? A Content Analysis of the Twitter Use Made by Radio RAI and RNE. Communication E Society/Comunicación y Sociedad. 27 (2), 125-146.

Boumans, J. (2004). Cross-media. E-Content Report 8. Acten Project E-content Reports Content Market Monitor Newsletter. Recuperado de http://www.acten.net/cgibin/WebGUI/www/index.pl/newsletter 19 ? wid $=806$ yfunc $=$ viewSubmissionysid $=1238$

Cabrera, M. A. (2016). La innovación: concepto y taxomización. En Sábada, Ch., García, J. A., \& Martínez-Costa, M. P. (Coords.). Innovación y desarrollo de los cibermedios en España. (pp. 23-30). Editoria EUNSA: España.

Campos-Freire, Francisco (2015). Adaptación de los medios tradicionales a la innovación de los metamedios. El profesional de la información. 24(4): 441-450. DOI: http://dx.doi. org/10.3145/epi.2015.jul.11

Castelló, A. (2010). Estrategias empresariales en la Web 2.0. Alicante: ECU.

Centereno de Arce, M. J. (2016). La utilización de los medios sociales en los programas de referencia de la radio española. Tesis inédita de Doctorado. Universidad de Murcia. Murcia - España. Recuperada de http://www.tdx.cat/handle/10803/401812

Centro Nacional de Consultoría. (2016). Estudio continuo de audiencia radial ECAR tercera ola 2016. Bogotá - Colombia.

Clemons, E. K. (2009). The complex problem of monetizing virtual electronic social networks. Decision Support Systems. 48 (1), 46-56.

ComScore. (2015). Futuro Digital Colombia 2015. Recuperado de https://www.comscore.com/ lat/Prensa-y-Eventos/Presentaciones-y-libros-blancos/2015/Futuro-Digital-Colombia-2015

Chan, Y.; \& Ngai, E. (2011). Conceptualising electronic word of mouth activity: An inputprocess-output perspective. Marketing Intelligence and Planning. 29 (5), 488-516.

Contreras, G. (2017). Hábitos de usos de internet entre los colombianos: Entre APP competentes y APP dependientes. Revista RCT (75), 56-61. Recuperado de https://issuu.com/revistarct/docs/revista_rct75_final

Del Pino, Castelló \& Ramos Soler (2013). La comunicación en cambio constante: branded content, community management, comunicación 2.0, estrategia en medios sociales. Fragua: España

EBU (2016). Audience trends 2016. Public version. Intelligence Media Service. Recuperado de https://www.ebu.ch/files/live/sites/ebu/files/Publications/EBU-MIS\%20-\%20Radio\%20 Audience\%20Trends\%202016\%20(Public).pdf

Edmond, M. (2014). All the platforms considered: Contemporary radio and transmedia engagement. New Media \& Society. Recuperado de http://nms.sagepub.com/content/ear ly/2014/04/06/1461444814530245

Fontdevila, J. \& Lamelo, C. (2015). La influencia social 2.0 en los programas magazín de las 
radios generalistas españolas con relación a su audiencia. Estudios sobre el Mensaje Periodístico. 21 (2), 813-831. DOI: http://dx.doi.org/10.5209/rev_ESMP.2015.v21.n2.50886

González Molina, S. \& Ramos del Cano, F. (2013). El uso periodístico de Facebook y Twitter: un análisis comparativo de la experiencia europea. Historia y Comunicación. 18 (Especial Noviembre), 419-433. DOI: http://dx.doi.org/10.5209/rev_HICS.2013.v18.44253

Gutiérrez, M.; Ribes, X. y Monclús, B. (2011). La audiencia juvenil y el acceso a la radio musical de antena convencional a través de Internet. Comunicación y Sociedad. XXIV (2), 305-331.

Gutiérrez, M.; Martí, J. M.; Ferrer, I.; Monclús, B; \& Ribes, X. (2014). Los programas radiofónicos españoles de prime time en Facebook y Twitter: Sinergias entre la radio convencional y las redes sociales. Revista Latina de Comunicación Social. (69), 418-434. DOI:http:// dx.doi.org/10.4185/RLCS-2014-1018

Gutiérrez-Coba, L., Gómez, J. A., Salgado, A., Estrada, J., \& Ramírez, M. J. (2012). Criterios y hábitos de consumo de información periodística on-line en Colombia. El profesional de la información. 21 (3), 261-267. DOI: http://dx.doi.org/10.3145/epi.2012.may.06

Hennig-Thurau, T.; Malthouse, E.; Friege, Ch.; Gensler, S.; Lobschat, L.; Rangaswamy, A.; \& Skiera, B. (2010). The Impact of New Media on Customer Relationships. Journal of Service Research. 13 (3), 311-330.

Hernández, R.; Fernández, C.; \& Baptista, P. (2003). Metodología de la investigación. McGrawHill: México.

Herrera-Damas, S.; \& Requejo-Alemán, J. L. (2012). Spanish talk Radio Stations on Twitter. Still reluctant to embrace its potential. En Oliveira, M.; Portela, P.; \& Santos, L. A. (Eds). Radio Evolution: Conference Proceedings. (pp. 49-62). Braga: University of Minho. Communication and Society Research Centre.

Holsti, O. (1969). Content analysis in communication research. Free Press: Nueva York.

IAB Spain (2017). Estudio anual de redes sociales. Recuperado de http://iabspain.es/wp-content/ uploads/20170221_estudioaudioonline_vcorta.pdf

IBOPE (2017). Consumo de medios en Colombia. Recuperado de http://www.kantaribopemedia.com.co/

Jenkins, H. (2006). Convergence culture: Where old and new media collide. New York: New York University Press.

Lozano, J. C. (1994). Hacia la reconsideración del análisis de contenido en la investigación de los mensajes comunicacionales. En Cervantes, C.; \& Sánchez, E. (coords.). Investigar la comunicación: propuestas iberoamericanas. (pp. 135-157). ALAIC/CEIC: Guadalajara.

Macnamara, J. (2010). Remodelling media: the urgent search for new media business models. Media International Australia. 137, 20-35.

MD. Marketing directo (2017). 20 millones de españoles utilizan las redes sociales cada día con Facebook y Whatsapp como lideres. Recuperado de https://www.marketingdirecto.com/digital-general/ social-media-marketing/20-millones-espanoles-utilizan-redes-sociales-dia-facebook-whatsapplideres

Martí, J. M.; Monclús, B.; Gutiérrez, M.; \& Ribes, X. (2015). La radio, modelo de negocio en transición: estrategias de oferta y comercialización en el entorno digital. Quaderns del CAC. XVIII (41): 13-22. 
Martínez-Costa, M. P. (2015). Radio y nuevas narrativas: de la crossradio a la transradio. En, Oliveira, M. \& Ribeiro, F. (Eds.). Radio, sound and Internet Proceedings of Net Station International Conference. (pp. 168-187). Centro de Estudos de Comunicação e Sociedade (CECS), Universidade do Minho: Brasil.

Mittel, J. (2006). Narrative Complexity in Contemporary American Television. The Velvet Light Trap, vol. 58. Recuperado de https://seguecommunity.middlebury.edu/view/html/site/ jmittell/node/4230077

Monclús, B.; Gutiérrez, M.; Ribes, X.; Ferrer, I.; \& Martí, J. M. (2015). Listeners, social Networks and the construction of Talk Radio Information's Discourse in the Age 2.0 Age. En Bonini, T. \& Monclús, B. (eds.). Radio Audiences and Participation in the Age of Network Society. (pp. 91-115). Routledge: New York.

Niqui, C. \& Segarra, L. (2016). Mapa de la radiodifusió analògica i digital a Catalunya. En Martí, J.M. \& Monclús, B. Informe 2012-2014 sobre la Ràdio a Catalunya: 11-61. Servei de Publicacions: Universitat Autònoma de Barcelona. Recuperado de http:/www.l-obsradio. cat/informe-anual-radio.html

OFCOM. (2015). News comsumption in the UK research rapport. Recuperado de https://www. ofcom.org.uk/_data/assets/pdf_file/0020/77222/News-2015-report.pdf

Ortega, F. \& Galhardi, C. (2013). Propuesta Metodológica para el análisis de contenido de la parrilla de Televisión en Brasil: Análisis de un caso práctico en los estados de São Paulo, Rio Grande do Sul, Estado da Bahía. Actas 2ํongreso Nacional sobre Metodología de la Investigación en Comunicación: Investigar la Comunicación hoy. Revisión de políticas científicas y aportaciones metodológicas. Recuperado de http:/uvadoc.uva.es/ handle/10324/3038

Pinseler, J. (2015). Domesticated Voices. Listener 'Participation' in Everyday Radio Shows. En Bonini, T. \& Monclús, B. (eds.). Radio Audiences and Participation in the Age of Network Society. (pp. 56-71). Routledge: New York.

Piñeiro Otero, T. (2015). De las ondas a los 140 caracteres. El uso de Twitter por los principales programas de la radio española. Palabra Clave. 18 (3), 815-841. DOI: http://dx.doi. org/10.5294/pacla.2015.18.3.8

RAJAR (2016). Rajar Data Infographic. Recuperado de http://www.rajar.co.uk/docs/news/ RAJAR_DataRelease_InfographicQ42016.pdf

Ramos del Cano, F. (2014). Redes sociales y participación radiofónica: análisis de caso de Twitter y Facebook en la Cadena SER. Ámbitos. 25. Recuperado de http://ambitoscomunicacion.com/2014/ redes-sociales-y-participacion-radiofonica-analisis-de-caso-de-twitter-y-facebook-en-la-cadenaser/

Redondo, M. (2007). Un análisis de contenido dual. Propuesta metodológica para el estudio de internet como fuente. Empiria. Revista de metodología de las Ciencias Sociales. (13), 35-58. DOI: http://dx.doi.org/10.5944/empiria.13.2007.1158

Reis, A. I. (2015). O àudio nas cibernotícias das ràdios. Editora Media XXI: Porto - Portugal.

Ribes, X., Monclús, B. \& Gutiérrez, M. (2015). Del oyente al radioprosumer: gestión de la participación de la audiencia en la radio del siglo XXI. Trípodos. 36. 55-74. 
Stark, B.; \& Weichselbaum, P. (2013). What attracts listeners to Web radio? A case study from Germany. The Radio Journal- International Studies Broadcas \& Audio Media. 11 (2), 185-202. DOI: http://dx.doi.org/10.1386/rjao.11.2.185_1

Vara-Miguel, A. \& Diaz-Espina, C. (2015). Nuevos modelos de negocio, creación de valor y el cambiante rol de la prensa en los sistemas democráticos. Tripodos. 36, 151-166.

Wimmer, R. \& Dominick, J. (1996). La investigación científica de los medios de comunicación. Una introducción a sus métodos. Barcelona: Bosch. 